\title{
The influence of relaxation with electromyography and Islamic prayer programs for female Muslim
}

\author{
Wang Jing ${ }^{\star 1}$ Muhanmmad Nubli Abdul Wahab ${ }^{1}$, Ahmed N Abdalla ${ }^{2}$, Gu Ming, Liu Yao ${ }^{1}$ and \\ Hanisah Mohd Noor ${ }^{4}$ \\ ${ }^{1}$ Faculty of Technology, University Malaysia Pahang, Malaysia. \\ ${ }^{2}$ Faculty of Electrical and Electronics Engineering, University Malaysia Pahang, Malaysia. \\ ${ }^{3}$ Faculty of Foreign Languages and Literature, Northwest University for Nationalities, China. \\ ${ }^{4}$ Department of Psychiatry, Kuliyyah of Medicine, International Islamic University Malaysia, Malaysia.
}

Accepted 20 January, 2012

\begin{abstract}
Chronic work-related stress can fray nerves, keep you up at night and contribute to significant and independent risk problems such as heart disease and depression. Particularly, chronic job strain can put both your physical and emotional health at risk. Muscle relaxation is a useful evidence based teaching and treatment approach for physical/remedial therapists. It can show unnecessary covert muscles co-contractions and the muscles staying tense after performing a task and not relaxing. The physiological monitoring can identify low level muscle activity which is not recognized by either the client or the therapist. Electromyography (EMG) allows the invisible muscle tension to become visible and is illustrated through a case study of a client who sought treatment because of shoulder pain. Her pain started when she became employed in a vegetable store and continued to increase to the point that she was afraid that she would have to stop working. During the assessment sessions, the trapezius and deltoid EMG were recorded while her role played her job task which is keyboard typewriting practice programs. The EMG showed increased shoulder tension, which did not return to baseline; there baseline was also an absence of regenerative EMG gaps. Salah is the physically demanding practice of formal prayer in Islam that helps adjust the rhythm of the body. After Salah training for clients, substantial upper trapezius muscles activity can be demonstrated. In the present investigation, the muscle activity of the right and left external upper trapezius muscles was examined to assess the level of muscle activity during Islamic prayer. The EMG showed more significant decrease and relaxation compared with non-training tasks of clients. After actually training using Salah with reduced dysponesis, the client reported a significant decrease in headache and less exhaustion after a day's work. At 1 month follow up, she reported a significant decrease in her shoulder complaints and was able to continue her job without shoulder problems. In summary, the findings are EMG-based. Salah augments the behavioral interventions because it helps develop internal awareness and enhance health status and reduce the risk of shoulder problems.
\end{abstract}

Key words: Islamic prayer, relaxation, electromyography, stress.

\section{INTRODUCTION}

Epidemiological studies have consistently demonstrated that chronic work-related stress is a significant and independent risk factor for cardiovascular and metabolic diseases and associated mortality (Chandola et al., 2006;

${ }^{*}$ Corresponding author. E-mail: ahmedras_76@yahoo.com
Kivimaki et al., 2006; Rosengren et al., 2004; Kivimaki et al., 2002). For example, a study enrolling over 10,000 participants concluded that individuals with high workrelated stress were more than twice as likely to develop metabolic syndrome versus those reporting lower levels of stress (Chandola et al., 2006). Studies have also noted a strong association between work-related stress and coronary artery disease (CAD) (Rosengren et al., 2004; 
Kivimaki et al., 2002) and a recent meta-analysis has concluded that work stress can increase the risk of myocardial infarction by $50 \%$ (Kivimaki et al., 2006). The link between stress and chronic disease is mediated by endocrine pathways of the sympathetic nervous system (SNS) including the hypothalamus-pituatary-adrenal (HPA) axis (Cohen et al., 2007; Rosmond and Björntorp 2000). Cortisol, the main effector of HPA activation, increases circulating fatty acid and glucose concentrations and inhibits insulin. This hormone mediated pathway prepares the body for physical exertion (that is, "fight or flight"). The actions of cortisol are particularly concerning in a sedentary work environment given that psychological stress compounded by inactivity induces hyperlipidemia and hyperglycemia, antecedents to more advanced cardio metabolic disease (for example, hypertension, obesity, type 2 diabetes, CAD, etc.) (McEwan, 1998; Vrijkotte et al., 1999; Siegrist et al., 1997). Interventions that holistically mitigate workrelated stress and inactivity may reduce the risk of chronic diseases.

Salah (Yucel, 2008) is a ritual prayer, having prescribed conditions, a prescribed procedure and prescribed times. Salah has been made compulsory on all believers in all situations. It is an activity which cannot be neglected under any circumstance. This obligation on us should not be regarded as a burden, but rather it should be cherished and appreciated. It is said that a pious person is one who enjoys their prayer. This statement conforms to the Hadith, which states that the prophets' three most beloved things were his wives, perfume and prayer. Besides attaining spiritual upliftment from performing one's salah, one may also gain many physical and psychological benefits (Childre and McCraty, 2001). The prophet Muhammad (SAW) is recorded in a Hadith (Ibn Majah) stating that prayer is a cure for many diseases. The wisdom in this claim can be understood when assessing the physical benefits of the act of prayer.

Salah, which is commonly used for muscle relaxation, can be performed by most people, including young people and cardiac patients. It has been used to build core stability during and after pregnancy and has been shown to increase creativity and reduce stress, as well as to improve muscle power, dexterity, visual perception and reaction time. However, while strength, endurance, and muscle reaction times have been previously quantified, little have been done to quantify muscle use during salah through the use of the electromyogram (EMG). The EMG, when measured by surface electrodes above an active muscle, represents an interference pattern giving the summation of activity of the underlying muscle fibers (Petrofsky and Lind, 1980). The amplitude of the surface EMG (sEMG) is generally related to the tension developed in muscle (Petrofsky et al., 1980; Petrofsky et al., 2005). Therefore, the EMG has proved to be a useful measure in assessing both the extent of muscle activity and muscle fatigue (Petrofsky and Lind, 1980; Petrofsky and Laymon, 2005; Bigland and Lippold 1954; Dahms et al., 1975; Lindstrom et al., 1970; Karlsson and Gerdle 2001). While some investigators find some variation in the EMG tension relationship due to the type of electrode (needle or surface) or the size or position of the electrodes (Alon, 1985; Alon et al., 1994), if the EMG is normalized as a percent of the muscle's maximum EMG during a maximum strength measurement, the EMG is a reliable tool to measure muscle use (Lindstrom et al., 1970; Karlsson and Gerdle, 2001).

This paper uses the EMG to quantify upper trapezius muscle activity during performing salah programs exercises versus performing the seated position to understand the magnitude of muscle use associated with salah after keyboard typewriting practice programs. This is particularly important since salah has been reported to assist in implementing practical interventions to enhance health status and reduce the risk of shoulder problems.

The main research questions and hypotheses are: Research question 1: Does samples' stress symptoms reduce and health situation improve with the incorporation of salah training for female Muslim compared with the control group? Null hypothesis $\left(\mathrm{H}_{\mathrm{o}}\right)$ : There is no improvement in samples' relaxation performance with the incorporation of salah training for women as measured by EMG signals. Alternate hypothesis $\left(\mathrm{H}_{1}\right)$ : samples' relaxation performance improves as measured by EMG signals with the incorporation of salah training for women. Research question 2: What are the participants' EMG and mental benefits from using the protocol?

\section{MATERIALS AND METHODS}

\section{Subjects}

The target population of participants was recruited through the student union, advertisements on the website and recommendations from associated professors in the University Malaysia Pahang. Respondents underwent a structured telephone interview and filled forms to ensure they met the criteria of irregular salah performances for female Muslim and were then randomly assigned to an intervention or control group. The subject was asked about age, nationality and physical situation. Questionnaires were administered to applicants who fulfilled the standard perceived stress scale (PSS) form (Cohen et al., 1988), which provides an idea of her general stress state.

From a total of 107 female volunteers with over 20 scores on the PSS forms, 23 eligible subjects were included in the study. The samples consisted of 23 healthy subjects, 16 female undergraduate students and 7 female graduate students $(6$ students with migration backgrounds, mean age 22.5 years $(S D=2.2)$; range 18 to 25$)$, mean height $=156.0 \mathrm{~cm}(\mathrm{SD}=5.2)$ and mean weight $=55.2 \mathrm{~kg}(\mathrm{SD}$ $=3.4$ ). To eliminate any selection bias, the model let the computer randomly assign subjects to one of the two groups. People who met to pray for the prayer group were given just the names of the subjects and very preliminary information on their physical condition. Potential participants will call a research assistant who then screens them for eligibility as well as collecting basic demographic information. Demographic characteristics will be 
collected during initial screening, including country of birth language spoken in the campus, level of education, immigration background, regular menstrual cycle, praying frequency, if any government pensions/benefits are received, living situation and total time spent in a car as a driver or passenger each week. In addition, data on any medical conditions and medication use will be collected through the medical clearance forms participants fill out prior to commencing the study.

All subjects, belonging to either of the groups, signed a consent form, informing them of the possibility that they might or might not get salah training. So, none of the patients knew whether she actually got salah training and at the same time; every one stood an equal chance. Throughout, protecting the research participants' identities and privacy was of utmost importance. Thus, each participant was also asked to choose a pseudonym to protect her anonymity when beginning the interview process and was then referred to by her chosen pseudonym during the interview process. Subsequently, the researcher chose a second set of pseudonyms because of potential breach in anonymity.

According to their reports, they have good healthy conditions and no salah performance at least 7 days before training of the first session. To meet study inclusion/exclusion criteria for participation, the participants had no history of heart disease and musculoskeletal disorders, and were asked to avoid hard physical activity the day before the day of measurement, and the subjects were trained by their job task which is keyboard typewriting practice programs (Erik Peper et al., 2003). The subjects were training in order to work up their typing speed to over fifty words a minute after the whole week training. Inclusion criteria were: age between 18 and 25 years and had the good physical conditions, observed for at least 20 days. All subjects were informed of the study aim and the details of the academic and experimental examinations. Before participation, subjects gave their written informed consent. The study was approved by center for modern languages and human science at University Malaysia Pahang.

\section{Design and protocol}

The study employed a single-blind design. The experimenter performing the pre- and post-intervention measurements did not know to which group (intervention [11 samples) versus control (12 samples)] the subject belonged. The intervention group participated in 2-day sessions of attention EMG training led by a licensed psychologist. During Session 1 and 10, individual mindful salah was assessed by having the subject salah at various activities. The control group took part in the protocol in Session 1 and 10, without any prescribed treatment in between. In order to evaluate treatment efficacy regarding subjective and physiological outcomes, both groups took part in an extensive assessment protocol the week before and after intervention.

\section{Session 1}

Definition of devoutness during Islamic activities; relevance to stress and health; introduction to assigned texts; in-class writing in which students describe their hopes for and concerns about the course; practice salah; handouts for the next week's homework: readings, journal and practicing salah using $C D$ of the introduction to salah.

\section{Session 2}

Present detailed procedures during performing salah; conduct a detailed discussion about movements, postures and general meaning explanation of writings during salah.

\section{Session 3}

Paying attention to the importance of intention and discuss the importance and healthy benefits of salah; It is not necessary to say the intention with the tongue as long as a firm intention has been made in the heart. However, the explicit verbalization of this intention is not required, though it can be helpful. The person should think his prayer to be the last prayer so that he may perform the best he can. When making intention especially for salah, one must state the salah that one is making intention for. Muslims need to pay attention to the intentions for worship; introduce the evidence in the holy Quran and Hadith; introduce focus on pleasant moments; introduce some papers which present the healthy benefits when people adopt salah; introduce focus on painful moments anddiscuss coping with both emotional and physical pain.

\section{Session 4}

Introduce the further meaning and background of verse in Quran and Hadith as well as the writings inside salah the focus on these during salah for devoutness training.

\section{Session 5}

Introduce the standard way of Quran recitation and correct the basic faults during reciting. To be beneficial to relaxation with breathing, the standard reciting way is instructed to recite of Quran but alert the long tone having a comparatively great duration in order to benefit for breathing.

\section{Session 6}

Present the gentle of movements and reciting writings.

\section{Session 7}

Do the exercise of breathing paralleled with Quran and writings recitation.

\section{Session 8}

Introduce basically inspiring imaginations for the further meaning of recitation.

\section{Session 9}

Use interactive explanation for improving the performance in salah.

\section{Session 10}

Introduce and discuss interrelationship of stress and the immune systems; encourage them to performing devout salah in every cycle during salah.

\section{Electromyography}

The Myoscan-Pro (SA9401M-60) surface EMG sensor consists of 3 snap style receptacles - 2 active (positive and negative) and 1 reference (ground). This EMG sensor kit (T9401M-60) is for use with "Thought Technology ProComp Plus, ProComp Infiniti, 
ProComp 2 and Procomp5". It includes cable that connects the sensor head to the ProComp Infiniti encoder and snap lead cable. It is operated in $60 \mathrm{~Hz}$ environment. The sEMG has a positive, negative and a ground sensor. The signal is measured between the positive and negative. The ground acts as a reference point, and is used in filtering out electrical "noise" that is picked up from all three sensors. Whenever possible, we recommend the use of disposable sensors. sEMG measures muscle activity by detecting and amplifying the tiny electrical impulses that are generated by muscle fibers when they contract. Since all the muscle fibers within the viewing and recording area of the sensor contract at different rates, the signal detected by the sensor is a constantly varying difference of potential between its positive and negative sensors. The number of muscle fibers that are recruited during any given contraction depends on the force required to perform the movement. Because of this, the intensity (amplitude) of the resulting electrical signal is in proportional to the strength of contraction. When placing the sEMG sensor on the muscle site, the best reading are obtained when the sensor is placed on the muscle belly and its positive and negative sensors are parallel to the muscle fibers. When using the extender cable, though, the direction of the fibers becomes irrelevant since the electrodes are frequently placed on different muscle groups. The positive and negative sensors should still be placed on the muscle bellies. The reference electrode is best placed on a relatively neutral site, like a bony prominence, preferably at an equal distance from the other two sensors.

\section{Measurement of strength of the upper trapezius}

Evaluation of this complex signal by sEMG techniques may be divided into three types: (a) static, (b) dynamic, and (c) a combination of these two. The data produced by these techniques is markedly different. Thus, today, understanding EMG evaluation involves understanding the purpose of the assessment, the conditions under which the assessment was conducted, and how these conditions may influence the evaluation procedure. The activity of the muscle while at rest (that is, sitting) is studied in this evaluation procedure. This examination is usually conducted to determine which muscles are hyperactive. One or more muscles may be evaluated. This information is then utilized to direct relaxation training. Isometric strength of the upper trapezius muscles was measured in the seated position.

The order of the test was randomized among the subjects to minimize crossover effects. The participants were asked to avoid hard physical activities the day before the day of measurement. For measuring EMG, during the lunch time but before the second time salah (Zuhur), subjects were trained by their job task which is keyboard typewriting practice programs. The subjects were enrolled, if they worked up her typing speed to over seventy words a minute after the whole week training. Before the keyboard typewriting practice test started, the subject was told that if he finished the test within a certain amount of time, he would get a reward. If he would not manage to finish within this amount of time, he would be punished. After finishing the present-winning test, the subject was told that he had completed the task well enough to receive the present. During the subjects being tested, a sitting static trial was collected prior to the test conditions for later determination of periods of muscle activation. They were instructed to keep their stomach in, chest out, shoulder back and head out, in the same time, keep their eyes wide open with a fixed gaze on the screen. This test functioned as a reference test to induce mental and psychosocial stress. The test was done under time pressure. The subject had $20 \mathrm{~min}$ to complete 1000 words length keyboard typewriting programs. When an error was made, a red screen appeared, a buzzer sounded and the subject had to typewrite again. A countdown timer was running while the subject was red. Beeps sounded at 10:00,15:00 and 18:00 min. When time was almost up, the program started beeping every two seconds from 19:00 to 19:40 min and it beeped every second during the last $20 \mathrm{~s}$.

Afterwards, continuous EMG recordings were made using BioGraph Infiniti software. For salah intervention group, the experiments are designated by four periods of baseline, salah performing, post-recovery and post-baseline each as measuring periods. The periods were defined as follows: baseline $(3 \mathrm{~min}$ into the baseline period), performing nufl salah with two cycles (within 5 min, preparing ablution before stress tests), post-recovery (sitting on a comfortable armchair with 3 min after performing salah) and post-baseline ( 3 min after post-recovery). For the control group, the samples are instructed by instructor for 14 min relaxation with sitting on a comfortable armchair with paced breathing. In this group, biometric indicators of EMG are measured by the same periods as the salah intervention group. The fellow-up session is designed by measuring the long-term effects of two groups. The measures were administered 4 weeks after the last treatment session. The experimental groups completed the stress tests. The modified PSS form of 2 days was filled out after each stress test and stress test in the follow-up session.

\section{Devout breathing exercise}

The similar exercise was based on a script used in mindfulnessbased cognitive therapy (MBCT) (Segal et al., 2002) and in a laboratory study by Arch and Craske (2006). Participants are guided to become aware of physical sensations especially those associated with the process of breathing and to observe them without the intention of altering them. Participants are asked to notice in an accepting, non-judgmental manner when their minds wander to something other than the exercise and to gently return focus to the sensations of breathing when this occurs. This basic salah exercise embodies the central features of mindfulness practice: intentionally paying attention to moment-by-moment experience with an attitude of acceptance (Arch and Craske, 2006; Kabat-Zinn, 1994). However, to reduce potential demand characteristics in self-reporting of decentering, instructions did not include language or techniques applied in MBCT ((Arch and Craske, 2006) and other interventions that explicitly addressed viewing specific thoughts from an objective, decentered perspective. Such techniques include the use of metaphors (that is, imagining thoughts are images projected on a movie screen), labeling thoughts (that is, encouraging participants to label thoughts as worries, self-criticisms, etc.), or explicitly describing the idea of decentering (for example, encouraging participants to view thoughts as "just thoughts" and not objective reality or a reflection of one's true self). In contrast to these approaches, the primary focus of this exercise was on the direct perception of breathing rather than thoughts themselves. During the 3 min of silence, participants were encouraged to continue with this practice.

\section{Procedures}

Participants randomized to the experimental condition will engage in a 4-week salah program delivered at their place of work (University Malaysia Pahang, Cambang Campus). The salah sessions will be group-based, prescribed four times per week during lunch hour (approximately 30 min per session), and will be led by an experienced salah instructor from Islamic Center in University Malaysia Pahang. The programs will involve teaching beginner students safely and progressively over 2 weeks worshipping act which incorporates poses and postures, recitation of Quranic verses, breathing control and Dhikr.

The sequence design will be aimed at developing strength, flexibility, cardiovascular fitness and the ability to deal with stress. 
Table 1. Comparison of different levels of PSS scores in experimental groups.

\begin{tabular}{|c|c|c|c|c|c|c|}
\hline \multirow{3}{*}{ PSS score } & \multicolumn{3}{|c|}{ Salah intervention group } & \multicolumn{3}{|c|}{ Control group } \\
\hline & Before salah & After salah & Follow-up & Before training & After training & Follow-up \\
\hline & \multicolumn{6}{|c|}{ Sample population } \\
\hline$\geq 20$ & 0 & 4 & 5 & 0 & 0 & 0 \\
\hline 21 to 25 & 5 & 4 & 3 & 6 & 7 & 5 \\
\hline 26 to 30 & 4 & 3 & 3 & 4 & 4 & 4 \\
\hline$\leq 30$ & 2 & 0 & 0 & 2 & 1 & 3 \\
\hline Mean (SD) & $\begin{array}{c}25.35 \\
(3.9)\end{array}$ & $\begin{array}{c}20.55 \\
(5.7)\end{array}$ & $\begin{array}{c}19.41 \\
(6.9)\end{array}$ & $\begin{array}{c}26.77 \\
(4.1)\end{array}$ & $\begin{array}{c}25.19 \\
(4.7)\end{array}$ & $\begin{array}{r}26.21 \\
(5.0)\end{array}$ \\
\hline$P$ value & & 0.07 & & & 0.75 & \\
\hline
\end{tabular}

Many postures incorporate a simple and a more challenging version so participants will be able to choose the level of difficulty that is appropriate to them on any given day. Throughout sessions, participants were guided through standard and basic salah procedure performing and devoutness training. In the first part, a detailed procedure of performing salah was introduced including movements, postures and general meaning explanation of writings during salah. Afterwards, in the second part, the way of performing devout salah is introduced by Islamic coach including the importance of intention, the importance of salah, the further meaning and background of verse in Quran and Hadith as well as the writings inside salah, the standard way of Quran recitation, the gentle of movement and reciting writings, the exercise of breathing paralleled with Quran and writings recitation and inspiring imaginations for the further meaning of recitation. In detail, participants were taught standard salah procedure, which is $45 \mathrm{~min}$ exercise in which participants bring attention to different movements and postures by themselves and observe the sensations in those parts of the body, while trying to understanding general meaning explanation of writings during performing salah. The importance of intention is also a focal point of devoutnessbased salah training, which refers to the firm intent of the heart. Participants were instructed to understand the further meaning and background of verse in Quran and Hadith as well as the writings, and focus on the meaning inside salah. When a person begins thinking about something other than the contents, she is asked to gently bring herself back to the meaning inside salah. Participants were instructed to recite Quran by the standard way but alert the long tone having a comparatively great duration in order to benefit for breathing. When a person recites the holy Quran and writings in the fast ways other than the gentle and standard methods, she is asked to gently recite and exercise breathing way paralleled with Quran and writings recitation due to the standard recitation way producing the natural way for breathing training. Finally, participants were guided through inspiring imaginations for the further meaning of recitation which emphasize gentle paying attention to the literal meaning and then in-depth interpretation and allow participants to focus on the sensations in the body while performing salah. The instructor encouraged participants to eventually wean themselves off the compact disc recordings and guideline books through continued practice. Participants were asked to practice outside of group sessions for 5 times salah per day in the whole week. In the group meetings, participants had the opportunity to discuss their personal practice of devoutness-based salah.

All participants were advised to change into appropriate clothing prior to arriving at the venue. EMG was then measured during this activity. In control group, participants randomized to the control condition were given education about the benefits of physical activity and relaxation training but received no specific instructions about salah practice for the 4 weeks intervention period. All outcome measures were collected at baseline (week 0) and after the intervention period (week 4), as well as at 1 month follow-up. Qualified and experienced personnel, blinded to the group assignment of participants assess the primary and secondary outcomes within one testing session conducted at all three assessment time points. To normalize the EMG on both sets of experimental conditions, after lunch time, all clients were evaluated by job tasks which are keyboard typewriting practice programs in order to determine the maximum strength of upper trapezius.

\section{STATISTICAL ANALYSES}

Primary analysis was via intention-to-treat with all participants included regardless of dropout or level of adherence. Missing data were imputed according to the maximum likelihood expectation algorithm via the statistical package for the social sciences (IBMC, SPSS version19.0). Data were presented as the mean \pm standard deviation or median and range as appropriate. Confidence intervals were used to express group differences.

In this study, we examined effects of salah on PSS in women referred to salah clinic. Symptoms of stress in both groups were assessed and compared pre- and post- intervention and between the experimental and control groups. As the study was done in women's salah clinic, all subjects were female. Experimental and control groups consisted of 11 and 12 women, respectively. Comparison of educational states in both groups did not show any statistically significant difference between the groups. Mean PSS scores in the experimental group before salah was 25.35. This decreased to 20.55 after salah intervention. Furthermore, this decrease was statistically significant $(P=0.07)$ (Table 1$)$. Mean PSS scores in the control group was 26.77 at the beginning and 25.19 after training sessions, but these were also not significantly different ( $P$ value $=0.25$ ). From the whole training, this decrease in EMG was essentially maintained throughout the 22 days salah intervention and the variant trend maintained sable in follow-up in comparison with last two session. Moreover, the follow-up session shown that in baseline and post-baseline of the control group, the quantitative values of EMG of conservatively were unvarying compared with the baseline. When comparing the average prevalence of stress between the experimental and control groups, salah intervention group showed significant difference $(P$ value $=0.05)$. Therefore, salah intervention group essentially reduced throughout the subsequent sessions of intervention and follow-up in stress scores 
Table 2. Baseline and post-baseline comparison of EMG in experimental groups.

\begin{tabular}{lcccccc}
\hline \multirow{2}{*}{ Mean (SD) } & \multicolumn{3}{c}{ Salah intervention group } & \multicolumn{3}{c}{ Control group } \\
\cline { 2 - 7 } & Before salah & After salah & P value & Before training & After training & P value \\
\hline \multirow{2}{*}{ EMG (baseline) } & 12.61 & 7.97 & \multirow{2}{*}{0.03} & 11.97 & 12.32 & \multirow{2}{*}{0.97} \\
& $(2.37)$ & $(2.01)$ & & $(2.09)$ & $(2.16)$ & \\
\multirow{2}{*}{ EMG (post-baseline) } & 2.90 & 1.91 & \multirow{2}{*}{0.04} & 4.24 & 4.91 & \multirow{2}{*}{0.93} \\
\hline
\end{tabular}

prevalence at the beginning of the study.

From Table 2, mean EMG score before salah was 12.61 in the experimental group. This decreased to 7.97 post salah interventions in the baseline setting, and mean EMG score before salah was 2.90 in the experimental group. This decreased to 1.91 post salah interventions in the post-baseline setting, which represented a statistically significant difference $(P$ value 0.03 and 0.04 , respectively). Mean EMG score in control group was 11.97 at the beginning and 12.32 after training in the baseline setting, and mean EMG score in control group was 4.24 at the beginning and 4.91 after training in the post-baseline setting, representing no significant difference ( $P$ value $=0.97$ and 0.93 , respectively).

\section{DISCUSSION}

It is known that salah is a good training technique for muscle relaxation. From a psychological standpoint, it also reduces anxiety. But salah also has been shown to decrease neurological reaction time and improve muscle strength and endurance of the expiratory and upper trapezius muscles. This, then, has important benefits in terms of expiratory disorders such as asthma. For salah, if there is increased muscle power and muscle dexterity, then the exercise itself must be using appreciable muscle strength. This is especially true for women who wish to build core stability during and after pregnancy. In the present investigation, considerable muscle activity was found for the rectus abdominis and exterior oblique muscles during salah exercise involving the core muscles of the body. Studies show that strengthening of the core muscles with sit-ups or other types of upper trapezius exercise contributes to increased functional abilities. When the shoulders and lower back muscles are strengthened using exercise machines, bowel and bladder programs in rehabilitation or health centers have been shown to be more effective. Further, if individuals are prone to falling, increasing core muscle stability increases the person's ability to maintain balance while reaching. Thus, exercise of the core muscles increases overall aerobic capacity and improves stability of the upper and lower body through training of the upper trapezius muscles, resulting in improved therapeutic reach and balance, better breathing, and better activity of the bowel. One particularly interesting finding of this investigation was the fact that even for the rectus shoulders muscle, which is somewhat active during shoulders; the total muscle activity was substantially higher during the salah breathing programs than during sitting positions. Thus, salah breathing is not only efficient when exercising the rectus abdominis but it also provides a good workout for the oblique muscles as well. If, for example, a salah breathing workout were to continue for 10 breaths, this would be more relaxed. Sitting for rest are usually considered the gold standard and used quite commonly during relaxation civilian fitness programs. However, salah breathing programs, in contrast, are used less frequently but workloads are much higher. Therefore, salah breathing programs, when combined with other exercise modalities, provide a much better workout for the core muscles in the body. Considering the relaxation work with salah breathing programs, total body workouts would be much more time efficient if salah breathing exercises were combined and interlaced with other exercise modalities for a more efficient fitness program. Finally, since breathing exercises can be accomplished from the seated position, salah is more approachable and compliance will be better for people who have difficulty working in a supine position such as the elderly and disabled or people with obesity.

During the salah, every muscle in the body contracts isotnically and others isometrically. This gentle exercise also enhances endurance and diminishes tiredness. It helps the incapacitated to make the most of their remnant capabilities. The blood flow in strung muscle is low. During the prayers, the blood flow is greatly increased to the muscles. Blood flow sometimes increases even before the start of salah, with just the thought of performing the salah. In addition to needing fats, proteins and carbohydrates, the human body requires minerals such as potassium for nerve and muscle activity. Potassium deficiency leads to muscular and neurologic disorders. Potassium is also important in the transmission of nerve impulses and is a major positive ion in intracellular fluids. Potassium is involved in cellular enzyme activities, and it helps regulate the chemical reactions by which carbohydrates are converted to energy and amino acids are converted to protein. Also, potassium ions cause vasodilatation of arterioles. During salah, systolic blood pressure (BP) may rise (the larger number in BP) a little and the diastolic BP (the lower number in BP) may remain unchanged or even fall. 
However, after the salah are over, the BP may drop to just below normal levels which is a welcome sign. Salah improve respiratory efficiency; circulation in the capillaries surrounding the alveoli, or air sacs, is increased, and this brings about enhanced gas exchange and deeper breathing. The increase in the maximal consumption of oxygen is what makes the salah feel better. Those who perform salah, apart from the prescribed salah, are more alert and active than those who do not perform salah, even after the age of retirement. Salah improve physical strength and joint stability and reduce the risk of injury to the tendons and connective tissues. After age 40, the bone mineral density falls with age. Salah increase bone mineral density in both menopausal and in elderly women and prevents osteoporosis and maintains normalcy in bone structures. Osteoporosis results in hip fractures in women after menopause and in elderly men. The risk of osteoporosis is substantially reduced by regular salah prayers. The prayers improve lubrication of joints, movement and maintain flexibility. Deep vein thrombosis (the most common cause of ulceration of the legs in the elderly) is prevented by performing salah.

Salah, which is recitation of the Quran in different postures including deep bowing and prostration, is designed in such a way that it gives myriad benefits in all the three dimensions of life namely spiritual, mental and physical. The wisdom of salah in Islam is firstly to create a spiritual connection between man and God and secondly to relieve the person's mind completely from life's stress or issues by taking a mental and physical break. The benefit of salah is felt right from the outset when one gets refreshed as he performs ablution by washing his face, hands and feet to rid himself from his physical impurities to begin his commune with his creator. Once in salah, when he begins the recitation of the words of God focusing his mind on the sublime meaning of the divine words, he feels a spiritual elevation and a heightened and lasting sense of God's conscienceness. The protocol confirms the usefulness of these shorttimely breaks - the average time of each salah is $15 \mathrm{~min}$ to completely turn the attention to focus away from the mental and physical patterns of stress and move towards something of neutral or positive value and the passive disregard for the normal thought that would arise. This helps in breaking the stress response of the body and the body begins to relax. Adding to this is the different postures in salah which further help the body and mind to relax as it gives an outlet for the energy produced by the body in the stress response.

The one common feature of all Salah practices examined in this review is the apparent ability to practice salah without adopting a specific system of spiritual or religious belief. However, the extent to which spirituality and belief are part of any given salah practice is poorly described. Furthermore, if the Taoist metaphysical assumptions of Qi Gong are crucial to successfully understand, visualize, and guide Qi, then at least this practice requires adopting a specific belief system. The extent to which spirituality or belief play a role in any salah practice appears to depend in large part on the individual practitioner. Though the traditional practices were developed within specific spiritual or religious contexts (Vipassana, Zen Buddhist meditation, Yoga, Tai Chi, Qi Gong), and therefore have spiritual or religious aspects, this does not mean that a practitioner must adopt the belief systems upon which they were based. In addition, some practices developed for purposes other than spiritual enlightenment; for example, Tai Chi and Qi Gong were developed within a system martial exercise and traditional Chinese medicine, respectively. Though Yoga, too, has spiritual and religious components, it is often considered more properly a system of metaphysics and psychology, especially when the ethical instructions are ignored. In summary, it appears that all salah practices can be performed, to some degree, without adopting a specific system of spirituality or belief.

\section{REFERENCES}

Alon G, Kantor G, Ho HS (1994). Effects of electrode size on basic excitatory responses and on selected stimulus parameters. J Orthop. Sports Phys. Ther., 20: 29-35.

Alon G (1985). Effects of electrode size on basic excitatory responsesHigh voltage stimulation. Phys. Ther., 65: 890-895.

Arch J, Craske M (2006). Mechanisms of mindfulness: emotion regulation following a focused breathing induction. Behaviour Res. Therapy, 44: 1849-1858.

Bigland B, Lippold O (1954). The relation between force, velocity and integrated EMG. J. Physiol., 123: 214-224.

Chandola T, Brunner E, Marmot M (2006). Chronic stress at work and the metabolic syndrome: prospective study. British Med. J., 332(7540): 521-525.

Childre D, McCraty R (2001). Psychophysiological Correlates of Spiritual Experience. Biofeedback, 29(4): 13-17.

Cohen S, Janicki-Deverts D, Miller G (2007). Psychol. Stress Disease. JAMA, 298(14): 1685-1687.

Cohen S, Williamson G, Spacapan S, Oskamp S (1988). Perceived Stress in a Probability Sample of the United States. The SocialPsychology of Health, Newbury Park, CA: Sage.

Dahms TE, Petrofsky JS, Lind AR (1975). Relationship between blood flow and muscle tension at low levels of muscular performance. Fed. Proc., 34: 370.

Erik Peper, Vietta S, Wilson, Katherine H, Gibney,Kate Huber, Richard $H$, Dianne M, Shumay (2003). The Integration of Electromyography (SEMG) at the Workstation: Assessment, Treatment, and Prevention of Repetitive Strain Injury (RSI). Appl. Psychophysiol. Biofeedback, 28(2): 167-182.

Kabat-Zinn J (1994). Wherever you go there you are: Mindfulness meditation in everyday life. New York: Hyperion.

Karlsson S, Gerdle B (2001) Mean frequency and signal amplitude of the surface EMG of the quadriceps muscles increase with increasing torque-a study using the continuous wavelet transform. J. Electromyogr. Kinesiol., 11: 131-140.

Kivimaki M, Leino-Arjas $\mathrm{P}$, Luukkonen R, Riihimaki $\mathrm{H}$, Vahtera J, Kirjonen J (2002). Work stress and risk of cardiovascular mortality: prospective cohort study of industrial employees. British Med. J., 325: 857.

Kivimaki $M$, Virtanen $M$, Elovainio $M$, Kouvonen $A$, Vaananen $A$, Vahtera $J$ (2006). Work stress in the etiology of coronary heart disease: a meta-analysis. Scandinavian J. Environ. Health, 32(6): 431-442.

Lindstrom L, Magnusson R, Petersen I (1970). Muscular fatigue and action potential conduction velocity changes studied with frequency 
analysis of EMG signals. Electromyography, 10:341-356.

McEwan B (1998) Protective and damaging effects of stress mediators. New England J. Med., 338(3): 171-179.

Petrofsky JS, Laymon M (2005) The influence of intramuscular temperature on surface EMG variables during isometric contractions. Basic Appl. Myology., 15: 61-74.

Petrofsky JS, Lind AR (1980) The influence of temperature on the amplitude and frequency components of the EMG during brief and sustained isometric contractions. Eur. J. Appl. Physiol., 44: 198-200.

Rosengren A, Hawken S, Ounpuu S, Sliwa K, Zubaid M, Almahmeed W, Blackett K, Sitti-amorn C, Sato H, Yuself S (2004) Association of psychosocial risk factors with risk of acute myocardial infarction in 11,119 cases and 13,648 controls from 52 countries (the INTERHEART study): case-control study. Lancet, 364: 953-962.

Rosmond R, Björntorp P (2000). The hypothalamic-pituitary-adrenal axis activity as a predictor of cardiovascular disease, type 2 diabetes and stroke. J. Int. Med., 247(2): 188-197.

Segal ZV, Williams JMG, Teasdale JD (2002). Mindfulness-based cognitive therapy for depression: A new approach to preventing relapse. NY: Guilford Press.
Siegrist J, Peter R, Cremer P, Seidel D (1997) Chronic work stress is associated with atherogenic lipids and elevated fibrinogen in middleaged men. J. Internal Med., 242: 149-156.

Vrijkotte T, van Doornen L, De Geus E (1999) Work stress and metaboilc and hemostatic risk factors. Psychosomatic Med., 61: 796805.

Yucel S (2008). The Effects of Prayer on Muslim Patients' Well Being Doctoral Dissertation. Boston University of School Teology, Boston, USA. Retrieved from ProQuest Database, UMI Number 330102. 